\title{
Integral equation theory of polydisperse colloidal suspensions using orthogonal polynomial expansions
}

\author{
F. Lado \\ Department of Physics, North Carolina State University, Raleigh, North Carolina 27695-8202
}

(Received 28 March 1996)

\begin{abstract}
A procedure is described for the calculation of the generalized pair distribution function $g\left(r, \sigma, \sigma^{\prime}\right)$, where $\sigma$ is a molecular random variable with distribution $f(\sigma)$, using generalized integral equations familiar from simple liquid theory. The method is based on expansions of all $\sigma$-dependent functions in the orthogonal polynomials $p_{j}(\sigma)$ associated with the weight $f(\sigma)$ and is computationally efficient. To illustrate the procedure, calculations are made for a charge-stabilized, polydisperse colloidal suspension with Schulz distribution of diameters $\sigma$. The method can be immediately generalized to fluids with internal degrees of freedom, for which $f(\sigma)$ must itself be self-consistently determined. [S1063-651X(96)12610-6]
\end{abstract}

PACS number(s): 02.70.-c, 82.70.Dd, 61.20.Gy, 61.25.Em

\section{INTRODUCTION}

Colloidal particles are typically composed of some $10^{6}-10^{12}$ atoms; modeled as solid spheres, they are thus some $10^{2}-10^{4}$ times the size of atoms. Their density fluctuations in a suspension are detected by light and thermal neutron scattering. The intuitive feeling that a concentrated suspension of colloidal particles (stabilized against coagulation) might be viewed as a "mesoscopic liquid"' is supported by the characteristic short-range liquid structure seen in the scattering data [1]. The description of the structure of these liquidlike suspensions using the tools of simple atomic liquids is complicated primarily by the fact that the particles of any given suspension display a continuous distribution of sizes; i.e., they are inherently polydisperse [2,3].

The most common approach to this problem is to integrate out (at least conceptually) the molecular degrees of freedom of the suspending fluid, such as water, and describe the suspension as a collection of mesoscopic particles interacting pairwise through a simple effective potential, typically hard sphere for sterically stabilized suspensions and screened Coulomb for charge-stabilized suspensions (with attendant charge polydispersity). These models immediately lend themselves to theoretical methods developed for simple liquids, with the added feature of polydispersity. Thus, size polydispersity in hard sphere models has been well studied using the analytic solution of the Percus-Yevick approximation [4-6]; charge polydispersity has been approximately mapped onto the same model [7], while the mean spherical approximation (MSA) [8] and rescaled MSA [9] have been applied to monodisperse charged colloids. The most general theoretical method, applicable to polydisperse particles with any potential, is the numerical solution of familiar integral equations of simple liquids [10]. This paper is concerned with the last of these.

In applying integral equation theory to polydisperse systems, D'Aguanno and Klein [10] deal with the polydispersity by replacing the continuous distribution $f(\sigma)$ of particle diameters $\sigma$ with a histogram for a finite set of $n$ well-chosen diameters, so that a more tractable $n$-component mixture substitutes for the polydisperse system. "Well-chosen', here means that the histogram for the discrete $n$-component system reproduces the first $2 n$ moments of the continuous distribution, a constraint that is equivalent to Gaussian integration using the zeroes of the $n$th orthogonal polynomial associated with the weight function $f(\sigma)$. This method works very well for relatively narrow distributions, for which a small number of components suffices. For broader distributions requiring larger numbers of components to maintain numerical accuracy, the "mixture" method using $n(n+1) / 2$ distinct correlation functions becomes computationally costly. In this paper, we show how expansions in the same set of orthogonal polynomials as used for the Gaussian integration avoids the rapid increase in computational cost for larger $n$ while retaining the numerical accuracy of Gaussian quadrature.

\section{POLYDISPERSE COLLOIDAL SYSTEMS}

Let $f(\sigma)$ be the distribution of particle diameters $\sigma$ in a homogeneous assembly of $N$ polydisperse colloidal particles contained in volume $V$ at temperature $T$. The density of particles at point $\mathbf{r}$ with diameter $\sigma$ is then

$$
\rho^{(1)}(\mathbf{r}, \sigma)=\left\langle\sum_{i=1}^{N} \delta\left(\mathbf{r}-\mathbf{r}_{i}\right) \delta\left(\sigma-\sigma_{i}\right)\right\rangle=\rho f(\sigma),
$$

where $\rho=N / V$, while the two-body density is

$$
\begin{aligned}
\rho^{(2)} & \left(\mathbf{r}, \sigma, \mathbf{r}^{\prime}, \sigma^{\prime}\right) \\
& =\left\langle\sum_{i \neq j} \delta\left(\mathbf{r}-\mathbf{r}_{i}\right) \delta\left(\sigma-\sigma_{i}\right) \delta\left(\mathbf{r}^{\prime}-\mathbf{r}_{j}\right) \delta\left(\sigma^{\prime}-\sigma_{j}\right)\right\rangle \\
& =\rho^{2} f(\sigma) f\left(\sigma^{\prime}\right) g\left(\left|\mathbf{r}-\mathbf{r}^{\prime}\right|, \sigma, \sigma^{\prime}\right),
\end{aligned}
$$

which defines the pair distribution function $g\left(r, \sigma, \sigma^{\prime}\right)$. In these expressions, $\mathbf{r}_{i}$ is the location of particle $i$ and $\sigma_{i}$ its diameter; the brackets denote a canonical ensemble average.

Knowledge of the pair distribution function (PDF) yields the "measured" structure factor $S^{M}(k)$ determined from static light-scattering experiments $[1,11]$, 
$S^{M}(k)$

$$
=1+\rho \frac{\int d \sigma d \sigma^{\prime} f(\sigma) f\left(\sigma^{\prime}\right) b(k, \sigma) b\left(k, \sigma^{\prime}\right) \widetilde{h}\left(k, \sigma, \sigma^{\prime}\right)}{\int d \sigma f(\sigma) b^{2}(k, \sigma)},
$$

where $b(k, \sigma)$ is the form amplitude for spherical particles of diameter $\sigma$ and $\widetilde{h}\left(k, \sigma, \sigma^{\prime}\right)$ the Fourier transform of the pair correlation function $h=g-1$. Further, the PDF provides the colloidal internal energy $U$ and pressure $p$, in the form

$$
\begin{gathered}
\frac{\beta U}{N}=\frac{1}{2} \rho \int d \mathbf{r} d \sigma d \sigma^{\prime} f(\sigma) f\left(\sigma^{\prime}\right) g\left(r, \sigma, \sigma^{\prime}\right) \beta \phi\left(r, \sigma, \sigma^{\prime}\right), \\
\frac{\beta p}{\rho}=1-\frac{1}{6} \rho \int d \mathbf{r} d \sigma d \sigma^{\prime} f(\sigma) f\left(\sigma^{\prime}\right) g\left(r, \sigma, \sigma^{\prime}\right) r \\
\times \frac{d \beta \phi\left(r, \sigma, \sigma^{\prime}\right)}{d r},
\end{gathered}
$$

where $\phi\left(r, \sigma, \sigma^{\prime}\right)$ is the effective pair potential and $\beta=1 / k_{B} T$ the inverse temperature, with $k_{B}$ Boltzmann's constant. The isothermal compressibility $K_{T}$,

$$
\begin{aligned}
\frac{1}{\rho k_{B} T K_{T}} & =\left(\frac{\partial \beta p}{\partial \rho}\right)_{T} \\
& =1-\rho \int d \mathbf{r} d \sigma d \sigma^{\prime} f(\sigma) f\left(\sigma^{\prime}\right) c\left(r, \sigma, \sigma^{\prime}\right),
\end{aligned}
$$

is obtained from the related direct correlation function $c\left(r, \sigma, \sigma^{\prime}\right)$, defined below in terms of $g\left(r, \sigma, \sigma^{\prime}\right)$ by the Ornstein-Zernike (OZ) equation. Thus the colloidal PDF $g\left(r, \sigma, \sigma^{\prime}\right)$ is the essential quantity for both structure and thermodynamics.

In a straightforward generalization of simple liquid theory [12], the PDF is obtained from the combination of the Ornstein-Zernike equation,

$$
\begin{aligned}
h\left(r_{12}, \sigma_{1}, \sigma_{2}\right) \equiv & g\left(r_{12}, \sigma_{1}, \sigma_{2}\right)-1 \\
= & c\left(r_{12}, \sigma_{1}, \sigma_{2}\right)+\rho \int d \mathbf{r}_{3} d \sigma_{3} f\left(\sigma_{3}\right) \\
& \times h\left(r_{13}, \sigma_{1}, \sigma_{3}\right) c\left(r_{32}, \sigma_{3}, \sigma_{2}\right),
\end{aligned}
$$

and the closure relation,

$$
\begin{aligned}
h\left(r, \sigma_{1}, \sigma_{2}\right)= & \exp \left[-\beta \phi\left(r, \sigma_{1}, \sigma_{2}\right)+h\left(r, \sigma_{1}, \sigma_{2}\right)\right. \\
& \left.-c\left(r, \sigma_{1}, \sigma_{2}\right)+b\left(r, \sigma_{1}, \sigma_{2}\right)\right]-1 .
\end{aligned}
$$

Here, $b\left(r, \sigma_{1}, \sigma_{2}\right)$ is the so-called bridge function, which must be approximated. To solve these equations for $g\left(r, \sigma_{1}, \sigma_{2}\right)$, it is convenient to rewrite them in terms of the indirect correlation function $\gamma=h-c$ and deconvolute Eq. (7) using Fourier transforms; this yields

$$
\begin{aligned}
\widetilde{\gamma}\left(k, \sigma_{1}, \sigma_{2}\right)= & \rho \int_{0}^{\infty} d \sigma_{3} f\left(\sigma_{3}\right) \\
& \times\left[\widetilde{c}\left(k, \sigma_{1}, \sigma_{3}\right)+\widetilde{\gamma}\left(k, \sigma_{1}, \sigma_{3}\right)\right] \widetilde{c}\left(k, \sigma_{3}, \sigma_{2}\right)
\end{aligned}
$$

for Eq. (7) and

$$
\begin{aligned}
c\left(r, \sigma_{1}, \sigma_{2}\right)= & \exp \left[-\beta \phi\left(r, \sigma_{1}, \sigma_{2}\right)+\gamma\left(r, \sigma_{1}, \sigma_{2}\right)\right. \\
& \left.+b\left(r, \sigma_{1}, \sigma_{2}\right)\right]-1-\gamma\left(r, \sigma_{1}, \sigma_{2}\right)
\end{aligned}
$$

for Eq. (8), which are now to be solved iteratively for $\gamma\left(r, \sigma_{1}, \sigma_{2}\right)$ given an approximate bridge function $b\left(r, \sigma_{1}, \sigma_{2}\right)$. The PDF is then finally obtained as

$$
\begin{aligned}
g\left(r, \sigma_{1}, \sigma_{2}\right)= & \exp \left[-\beta \phi\left(r, \sigma_{1}, \sigma_{2}\right)+\gamma\left(r, \sigma_{1}, \sigma_{2}\right)\right. \\
& \left.+b\left(r, \sigma_{1}, \sigma_{2}\right)\right] .
\end{aligned}
$$

We remark for completeness that the Fourier transform of a spherically symmetric function such as $c\left(r, \sigma_{1}, \sigma_{2}\right)$ is computed as

$$
\widetilde{c}\left(k, \sigma_{1}, \sigma_{2}\right)=\frac{4 \pi}{k} \int_{0}^{\infty} d r r c\left(r, \sigma_{1}, \sigma_{2}\right) \sin k r,
$$

whose inverse is

$$
c\left(r, \sigma_{1}, \sigma_{2}\right)=\frac{1}{2 \pi^{2} r} \int_{0}^{\infty} d k k \widetilde{c}\left(k, \sigma_{1}, \sigma_{2}\right) \sin k r .
$$

Following D'Aguanno and Klein [10], the final integration over $\sigma_{3}$ in Eq. (9) could be handled with Gaussian quadrature. Let $p_{j}(\sigma), j=0,1,2, \ldots$, with $p_{0}(\sigma)=1$, be the set of orthonormal polynomials associated with the weight $f(\sigma)$ and interval $(0, \infty)$, so that

$$
\int_{0}^{\infty} d \sigma f(\sigma) p_{i}(\sigma) p_{j}(\sigma)=\delta_{i j}
$$

where $\delta_{i j}$ is the Kronecker delta. Then Gaussian quadrature based on the $n$ roots $\sigma_{k}$ of $p_{n}(\sigma)$ gives the rule

$$
\int_{0}^{\infty} d \sigma f(\sigma) y(\sigma) \approx \sum_{k=1}^{n} w_{k} y\left(\sigma_{k}\right)
$$

with [13]

$$
w_{k}=\frac{1}{\sum_{j=0}^{n-1} p_{j}^{2}\left(\sigma_{k}\right)} .
$$

Equation (15) is exact if $y(\sigma)$ is a polynomial of degree $2 n-1$ or less. Application of this rule to Eq. (9) produces a set of equations equivalent to those of an $n$-component mixture of particles of diameters $\sigma_{k}$ and concentration $w_{k}$,

$$
\begin{aligned}
\widetilde{\gamma}\left(k, \sigma_{k}, \sigma_{r}\right)= & \rho \sum_{m=1}^{n} w_{m}\left[\widetilde{c}\left(k, \sigma_{k}, \sigma_{m}\right)\right. \\
& \left.+\widetilde{\gamma}\left(k, \sigma_{k}, \sigma_{m}\right)\right] \widetilde{c}\left(k, \sigma_{m}, \sigma_{r}\right) .
\end{aligned}
$$


Gaussian integration is generally very efficient in numerical work. However, the number of distinct correlation functions that must be stored and manipulated with this approach grows quadratically with $n$, which rapidly increases the computational cost. In the next section we show how the same ingredients can be used while largely avoiding this increasing cost.

\section{ORTHOGONAL POLYNOMIAL EXPANSIONS}

The alternative to Eq. (17) is based on expansions of all functions of $\sigma$ in the orthonormal polynomials $p_{j}(\sigma)$. We assume the $p_{j}(\sigma)$ are complete, so that any function $y(\sigma)$ can be expanded in this set,

$$
y(\sigma)=\sum_{j=0}^{\infty} y_{j} p_{j}(\sigma)
$$

with the expansion coefficients determined as

$$
y_{j}=\int_{0}^{\infty} d \sigma f(\sigma) y(\sigma) p_{j}(\sigma),
$$

which follows from Eq. (14). The numerical (approximate) version of these equations using the $n$ roots of $p_{n}(\sigma)$ read

$$
\begin{gathered}
y\left(\sigma_{k}\right)=\sum_{j=0}^{n-1} y_{j} p_{j}\left(\sigma_{k}\right), \\
y_{j}=\sum_{k=1}^{n} w_{k} y\left(\sigma_{k}\right) p_{j}\left(\sigma_{k}\right) .
\end{gathered}
$$

Pair functions such as $\gamma\left(r, \sigma_{1}, \sigma_{2}\right)$ are similarly expanded as

$$
\gamma\left(r, \sigma_{1}, \sigma_{2}\right)=\sum_{i, j=0}^{\infty} \gamma_{i j}(r) p_{i}\left(\sigma_{1}\right) p_{j}\left(\sigma_{2}\right)
$$

with the inverse

$$
\gamma_{i j}(r)=\int d \sigma_{1} d \sigma_{2} f\left(\sigma_{1}\right) f\left(\sigma_{2}\right) \gamma\left(r, \sigma_{1}, \sigma_{2}\right) p_{i}\left(\sigma_{1}\right) p_{j}\left(\sigma_{2}\right)
$$

The numerical versions are

$$
\begin{gathered}
\gamma\left(r, \sigma_{k}, \sigma_{m}\right)=\sum_{i, j=0}^{n-1} \gamma_{i j}(r) p_{i}\left(\sigma_{k}\right) p_{j}\left(\sigma_{m}\right) \\
\gamma_{i j}(r)=\sum_{k, m=1}^{n} w_{k} w_{m} \gamma\left(r, \sigma_{k}, \sigma_{m}\right) p_{i}\left(\sigma_{k}\right) p_{j}\left(\sigma_{m}\right) .
\end{gathered}
$$

Similar expansions hold for the other pair functions and their Fourier transforms. The unknown now becomes the set of functions $\gamma_{i j}(r)$.

With these expansions, the OZ equation (9) becomes

$$
\widetilde{\gamma}_{i j}(k)=\rho \sum_{k}\left[\widetilde{c_{i k}}(k)+\widetilde{\gamma}_{i k}(k)\right] \widetilde{c}_{k j}(k),
$$

or, in matrix form,

$$
\widetilde{\Gamma}(k)=\rho[\widetilde{C}(k)+\widetilde{\Gamma}(k)] \widetilde{C}(k),
$$

which has the solution

$$
\widetilde{\Gamma}(k)=\rho \widetilde{C}(k) \widetilde{C}(k)[I-\rho \widetilde{C}(k)]^{-1} .
$$

In these equations $\widetilde{\Gamma}(k), \widetilde{C}(k)$ are symmetric matrices with elements $\widetilde{\gamma}_{i j}(k), \widetilde{c}_{i j}(k)$ and $I$ is the unit matrix.

With these developments, the iterative solution of Eqs. (9) and (10) for the unknowns $\gamma_{i j}(r)$ can be summarized as a sequence of four steps that make up one iteration:

(i) $\gamma_{i j}(r) \rightarrow c_{i j}(r)$ : Closure. Construct

$$
\gamma\left(r, \sigma_{1}, \sigma_{2}\right)=\sum_{i, j} \gamma_{i j}(r) p_{i}\left(\sigma_{1}\right) p_{j}\left(\sigma_{2}\right)
$$

from the current coefficients $\gamma_{i j}(r)$, as well as $\phi\left(r, \sigma_{1}, \sigma_{2}\right)$ and $b\left(r, \sigma_{1}, \sigma_{2}\right)$ from appropriate expressions. (In most approximations, the bridge function is implicitly defined by the functional form of the closure equation.) Then form

$$
\begin{aligned}
h\left(r, \sigma_{1}, \sigma_{2}\right)= & \exp \left[-\beta \phi\left(r, \sigma_{1}, \sigma_{2}\right)+\gamma\left(r, \sigma_{1}, \sigma_{2}\right)\right. \\
& \left.+b\left(r, \sigma_{1}, \sigma_{2}\right)\right]-1
\end{aligned}
$$

and numerically evaluate the coefficients

$$
h_{i j}(r)=\int d \sigma_{1} d \sigma_{2} f\left(\sigma_{1}\right) f\left(\sigma_{2}\right) h\left(r, \sigma_{1}, \sigma_{2}\right) p_{i}\left(\sigma_{1}\right) p_{j}\left(\sigma_{2}\right)
$$

using Eq. (23b) to get finally

$$
c_{i j}(r)=h_{i j}(r)-\gamma_{i j}(r) .
$$

(ii) $c_{i j}(r) \rightarrow \widetilde{c_{i j}}(k):$ Transforms. Evaluate the transforms

$$
\widetilde{c_{i j}}(k)=\frac{4 \pi}{k} \int_{0}^{\infty} d r r c_{i j}(r) \sin k r .
$$

(iii) $\widetilde{c}_{i j}(k) \rightarrow \widetilde{\gamma}_{i j}(k):$ OZ equation. Perform the matrix operations

$$
\widetilde{\Gamma}(k)=\rho \widetilde{C}(k) \widetilde{C}(k)[I-\rho \widetilde{C}(k)]^{-1}
$$

for the new $\widetilde{\gamma}_{i j}(k)$.

(iv) $\widetilde{\gamma}_{i j}(k) \rightarrow \gamma_{i j}(r)$ : Inverse transforms. Evaluate the inverse transforms

$$
\gamma_{i j}(r)=\frac{1}{2 \pi^{2} r} \int_{0}^{\infty} d k k \widetilde{\gamma}_{i j}(k) \sin k r
$$

This completes one iteration. The cycle (i)-(iv) is repeated until self-consistency of the $\gamma_{i j}(r)$ is achieved.

Other quantities can be expressed directly in terms of the computed coefficients. Thus, the number-number pair distribution function is

$$
g_{N N}(r) \equiv \int d \sigma_{1} d \sigma_{2} f\left(\sigma_{1}\right) f\left(\sigma_{2}\right) g\left(r, \sigma_{1}, \sigma_{2}\right)=g_{00}(r)
$$

while similarly the number-number structure factor is 


$$
\begin{aligned}
S_{N N}(k) \equiv & \int d \sigma_{1} d \sigma_{2}\left[f\left(\sigma_{1}\right) \delta\left(\sigma_{1}-\sigma_{2}\right)\right. \\
& \left.+\rho f\left(\sigma_{1}\right) f\left(\sigma_{2}\right) \tilde{h}\left(k, \sigma_{1}, \sigma_{2}\right)\right]=1+\rho \widetilde{h_{00}}(k)
\end{aligned}
$$

The measured structure factor, Eq. (3), depends on all the pair correlation function coefficients,

$$
S^{M}(k)=1+\rho \frac{\sum_{i, j} b_{i}(k) b_{j}(k) \tilde{h}_{i j}(k)}{\sum_{j} b_{j}^{2}(k)},
$$

where the $b_{j}(k)$ are the expansion coefficients of the form amplitude $b(k, \sigma)$.

Equations (4) and (5) for the energy and pressure can also be expanded in coefficients of $g\left(r, \sigma_{1}, \sigma_{2}\right)$ and $\phi\left(r, \sigma_{1}, \sigma_{2}\right)$ or evaluated directly using $g\left(r, \sigma_{1}, \sigma_{2}\right)$ from Eq. (11) and Gaussian quadrature. In fact, it is useful to compute these quantities both ways as an internal check. The inverse compressibility, Eq. (6), is immediately found as

$$
\frac{1}{\rho k_{B} T K_{T}}=1-\rho \widetilde{c_{00}}(0) .
$$

Finally, we show in the Appendix that the colloidal excess free energy $A$ can be expressed as

$$
\begin{aligned}
\frac{\beta A}{N}= & -\frac{1}{2} \rho \int d \mathbf{r}\left\{c_{00}(r)+\frac{1}{2} \sum_{i, j}\left[c_{i j}^{2}(r)-\gamma_{i j}^{2}(r)\right]\right\} \\
& -\frac{1}{2 \rho} \int \frac{d \mathbf{k}}{(2 \pi)^{3}}\{\operatorname{lndet}[I+\rho \widetilde{H}(k)]-\operatorname{tr}[\rho \widetilde{H}(k)]\} \\
& +\frac{1}{2} \rho \int d \mathbf{r} \int_{0}^{1} d \xi \sum_{i, j} g_{i j}(r ; \xi) \frac{\partial b_{i j}(r ; \xi)}{\partial \xi},
\end{aligned}
$$

where $\widetilde{H}(k)$ is the matrix with elements $\widetilde{h}_{i j}(k)$ and det and $\operatorname{tr}$ are the determinant and trace operations. The last term in Eq. (38) must be approximated. For the HNC equation of the next section, for example, it is neglected.

A numerical example will illustrate the practical advantage to be gained by expanding the unknowns in orthogonal ponynomials tailored to the distribution function $f(\sigma)$. Consider the function

$$
A(\sigma)=a \frac{\sigma^{2} e^{b \sigma}}{1+b \sigma}
$$

shown in Fig. 1, where $a$ and $b$ are constants defined in the next section for the shielded Coulomb potential. The expansion coefficients $A_{j}$ of this function for $n=4$ and $n=10$ were computed using Eq. (20b) and the renormalized associated Laguerre polynomials of the next section. The results are shown in Fig. 2; it is clear that the coefficients $A_{j}$ for $j>4$ are negligible. When the function $A(\sigma)$ is reconstructed using Eq. (20a), seen in Fig. 1, the more detailed results shown for $n=10$ are in practice obtainable from just the first four coefficients $A_{j}$. (It should be noted in Fig. 2 that these coincide for the $n=4$ and $n=10$ computations.) This means that the number of points $n$ in a numerical quadrature like Eq. (20b) or (23b) can be increased for greater accuracy

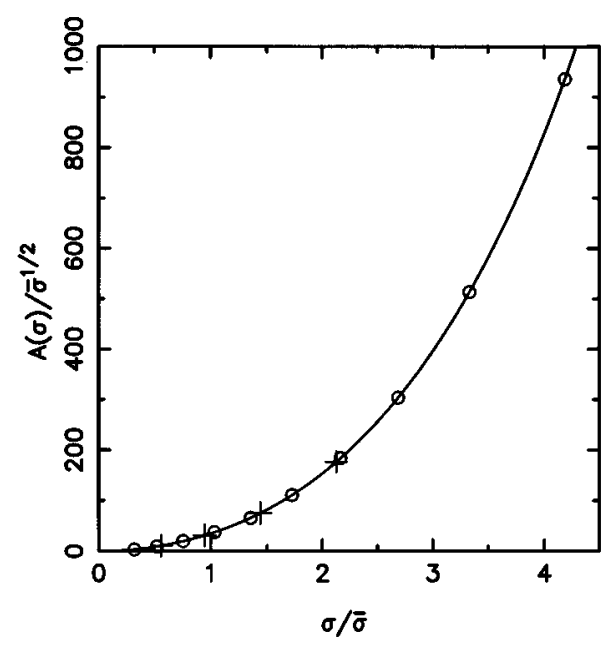

FIG. 1. The function $A(\sigma)$ from Eq. (46) (line) evaluated for $n=4$ (crosses) and $n=10$ (circles) at $s_{\sigma}=0.3$.

without the need for a proportionate increase in the number of coefficients used, thus unlinking the upper limits in the paired summations of Eqs. (20a) and (23a).

We will thus understand that while $n$-point integration is used in step (i) to numerically evaluate Eq. (29), the sums in Eq. (27) will be over $n^{\prime}$ terms, where $n^{\prime}<n$, and the matrices in step (iii) will correspondingly be $n^{\prime} \times n^{\prime}$ in size; $n^{\prime}$ will be determined empirically.

\section{RESULTS FOR THE SCHULZ DISTRIBUTION}

In this section, we present results obtained for chargestabilized, polydisperse colloidal suspensions using the orthogonal expansion method of Sec. III. The particle diameters $\sigma$ are taken to be distributed about a mean diameter $\bar{\sigma}$ according to the Schulz (gamma) distribution [14],

$$
f(\sigma)=\left(\frac{\alpha+1}{\bar{\sigma}}\right)^{\alpha+1} \frac{\sigma^{\alpha} e^{-(\alpha+1) \sigma / \bar{\sigma}}}{\Gamma(\alpha+1)}
$$

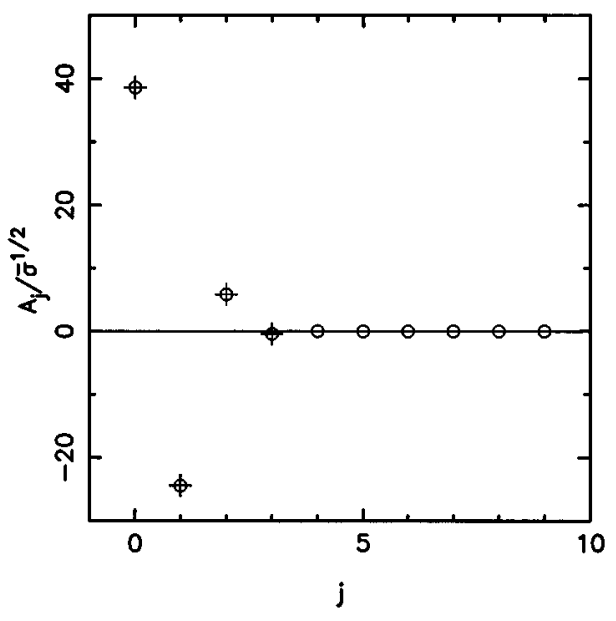

FIG. 2. Coefficients $A_{j}$ of the function $A(\sigma)$ from Fig. 1 for $n=4$ (crosses) and $n=10$ (circles). 
where $\Gamma(z)$ is the gamma function. The moments of this distribution are

$$
\left\langle\sigma^{j}\right\rangle=\frac{\Gamma(j+\alpha+1)}{\Gamma(\alpha+1)}\left(\frac{\bar{\sigma}}{\alpha+1}\right)^{j},
$$

and so the relative standard deviation is

$$
s_{\sigma}=\frac{\left(\left\langle\sigma^{2}\right\rangle-\langle\sigma\rangle^{2}\right)^{1 / 2}}{\langle\sigma\rangle}=\frac{1}{(\alpha+1)^{1 / 2}},
$$

which will be used to characterize the suspensions and define the parameter $\alpha$. The orthonormal polynomials for the Schulz distribution are

$$
p_{j}(\sigma)=\left[\frac{j ! \Gamma(\alpha+1)}{\Gamma(j+\alpha+1)}\right]^{1 / 2} L_{j}^{(\alpha)}\left((\alpha+1) \frac{\sigma}{\bar{\sigma}}\right),
$$

where the $L_{j}^{(\alpha)}(t)$ are the associated Laguerre polynomials.

The charged mesospheres are assumed to have a constant surface charge density, so that their charge polydispersity is mapped onto the size polydispersity according to

$$
Z(\sigma)=Z_{\bar{\sigma}}\left(\frac{\sigma}{\bar{\sigma}}\right)^{2}
$$

where $Z_{\bar{\sigma}}$ is the charge on a particle of mean diameter $\bar{\sigma}$, and to interact, beyond hard sphere contact, through a screened Coulomb potential,

$$
\beta \phi\left(r, \sigma_{1}, \sigma_{2}\right)= \begin{cases}\infty, & \text { if } r<\sigma_{12} \\ A\left(\sigma_{1}\right) A\left(\sigma_{2}\right) \exp (-\kappa r) / r, & \text { if } r>\sigma_{12},\end{cases}
$$

where $\sigma_{12}=\frac{1}{2}\left(\sigma_{1}+\sigma_{2}\right)$ and

$$
A(\sigma)=\frac{Z(\sigma) L_{B}^{1 / 2} \exp (\kappa \sigma / 2)}{1+\kappa \sigma / 2} .
$$

Here, $L_{B}=e^{2} / 4 \pi \epsilon_{0} \epsilon k_{B} T$ is the Bjerrum length and $\kappa=\left(4 \pi L_{B} \rho \bar{Z}\right)^{1 / 2}$ is the inverse Debye-Hückel screening length, with $\bar{Z}=Z_{\bar{\sigma}}\left(1+s_{\sigma}^{2}\right)$.

This is the same model studied by D'Aguanno and Klein [10]. To illustrate the polynomial expansion method, we have recalculated their results for the state with $\bar{\sigma}=250 \AA$, $Z_{\bar{\sigma}}=200$, and $L_{B}=7.01 \AA$, at the reduced density $\rho \bar{\sigma}^{3}=0.005$, using the hypernetted chain (HNC) [12] and Rogers-Young (RY) [15] closures,

$$
\begin{aligned}
g^{H N C}\left(r, \sigma_{1}, \sigma_{2}\right)= & \exp \left[-\beta \phi\left(r, \sigma_{1}, \sigma_{2}\right)+\gamma\left(r, \sigma_{1}, \sigma_{2}\right)\right], \\
g^{R Y}\left(r, \sigma_{1}, \sigma_{2}\right)= & \exp \left[-\beta \phi\left(r, \sigma_{1}, \sigma_{2}\right)\right] \\
& \times\left\{1+\frac{\exp \left[q(r) \gamma\left(r, \sigma_{1}, \sigma_{2}\right)\right]-1}{q(r)}\right\} .
\end{aligned}
$$

The RY closure features a mixing function,

$$
q(r)=1-e^{-\lambda r},
$$

TABLE I. Thermodynamics of a polydisperse colloidal suspension with shielded-Coulomb potential and Schulz distribution of relative standard deviation $s_{\sigma}$. Monte Carlo (MC) data are from D'Aguanno and Klein [10,16].

\begin{tabular}{lccccc}
\hline \hline & $s_{\sigma}$ & $\beta U / N$ & $\beta p / \rho-1$ & $\rho k_{B} T K_{T}$ & $\beta A / N$ \\
\hline MC & & 28.00 & 47.50 & & \\
RY & 0 & 28.30 & 47.78 & 0.00851 & \\
HNC & & 28.71 & 48.20 & 0.00999 & 31.91 \\
MC & & 27.66 & 47.14 & & \\
RY & 0.1 & 27.94 & 47.45 & 0.00854 & \\
HNC & & 28.35 & 47.87 & 0.01005 & 31.55 \\
MC & & 26.64 & 46.16 & & \\
RY & 0.2 & 26.89 & 46.48 & 0.00863 & \\
HNC & & 27.31 & 46.90 & 0.01025 & 30.49 \\
MC & & 24.97 & 44.50 & & \\
RY & 0.3 & 25.24 & 44.86 & 0.00879 & \\
HNC & & 25.67 & 45.30 & 0.01059 & 28.83 \\
RY & 0.4 & 23.12 & 42.65 & 0.00903 & \\
HNC & & 23.56 & 43.10 & 0.01111 & 26.68 \\
RY & 0.5 & 20.70 & 39.92 & 0.00939 & \\
HNC & & 21.15 & 40.39 & 0.01183 & 24.22 \\
\hline \hline
\end{tabular}

with a parameter $\lambda$ that is chosen to enforce consistency between the inverse compressibility gotten from Eq. (6) and that obtained by numerical differentiation of Eq. (5).

The computed thermodynamic values are shown in Table I for five Schulz distributions of increasing width, $s_{\sigma}=0.1$, $0.2,0.3,0.4$, and 0.5 , as well as the monodisperse case, $s_{\sigma}=0$, for reference. The present integral equation results are in good agreement with those found earlier $[10,16]$ for $s_{\sigma}$ up to 0.3 using the "mixture" method. Comparison with the Monte Carlo data of D'Aguanno and Klein [10,16], also shown in Table I, confirms that the RY approximation is producing the more reliable results, as is the case with monodisperse systems [15]. The number of coefficients $\gamma_{i j}(r)$ used in these calculations ranged up to 15 , corresponding to $n^{\prime} \leqslant 5\left(n^{\prime}=4\right.$ is adequate), while the Gaussian quadratures were carried out with $n \geqslant 7$ points. [In fact, because the polynomials are divergent for large $\sigma$, the smallest $n$ had to be used for the largest $s_{\sigma}$, since the diverging values of the $p_{j}(\sigma)$ led eventually to numerical divergence of the resummed $\gamma\left(r, \sigma_{1}, \sigma_{2}\right)$ in Eq. (27) for $\sigma$ values farther out in the wing.] The grids on the $r$ and $k$ axes were constructed with $N=1024$ points and intervals $\Delta r / \bar{\sigma}=0.1$ and $\Delta k=\pi / N \Delta r$.

Comparisons of the computed pair distribution and scattering functions with simulation have already been made by D'Aguanno and Klein $[10,11]$. Here we display some of the coefficients of these functions.

Local fluctuations in density and size may be expressed in normalized form as

$$
\delta \rho_{0}(\mathbf{r})=\frac{1}{\rho}\left(\sum_{j=1}^{N} \delta\left(\mathbf{r}-\mathbf{r}_{j}\right)-\rho\right),
$$

$$
\delta \rho_{1}(\mathbf{r})=\frac{1}{\bar{\sigma} \rho s_{\sigma}}\left(\sum_{j=1}^{N} \sigma_{j} \delta\left(\mathbf{r}-\mathbf{r}_{j}\right)-\bar{\sigma} \rho\right) .
$$




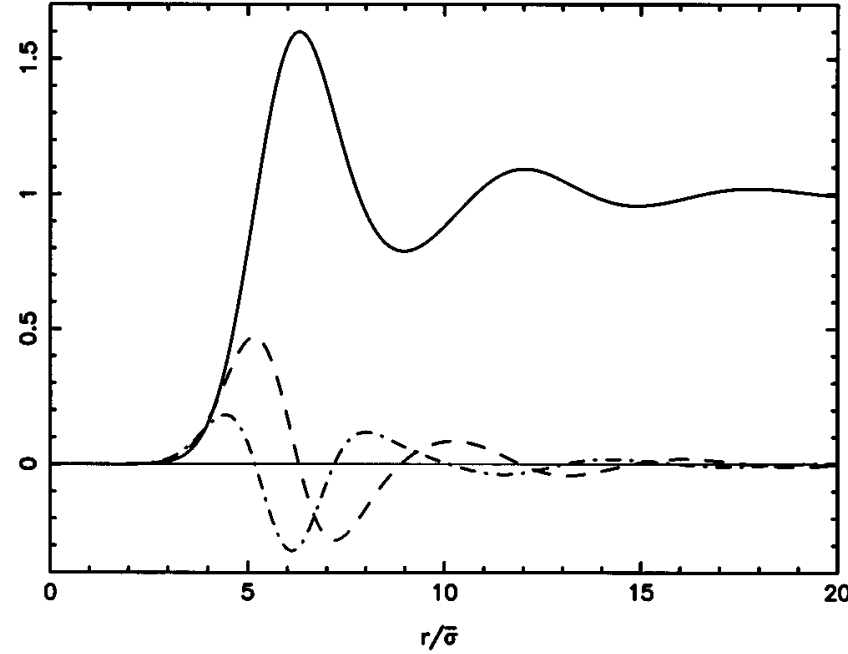

FIG. 3. Coefficients of the pair distribution function, $g\left(r, \sigma_{1}, \sigma_{2}\right)$, from the RY equation at $s_{\sigma}=0.3: g_{00}(r)$, solid line; $g_{10}(r)$, dashed line; $g_{11}(r)$, dash-dot line.

Their spatial correlations are then given by

$$
\begin{gathered}
\left\langle\delta \rho_{0}(\mathbf{r}) \delta \rho_{0}(\mathbf{0})\right\rangle=\frac{\delta(\mathbf{r})}{\rho}+\left[g_{00}(r)-1\right], \\
\left\langle\delta \rho_{1}(\mathbf{r}) \delta \rho_{0}(\mathbf{0})\right\rangle=-g_{10}(r), \\
\left\langle\delta \rho_{1}(\mathbf{r}) \delta \rho_{1}(\mathbf{0})\right\rangle=\frac{\delta(\mathbf{r})}{\rho}+g_{11}(r) .
\end{gathered}
$$

The functions $g_{00}(r), g_{10}(r)$, and $g_{11}(r)$ for these correlations are shown in Fig. 3 for the case with $s_{\sigma}=0.3$ calculated with the RY equation using $n^{\prime}=5$ and $n=10$. (The data in Figs. 1 and 2 are also for $s_{\sigma}=0.3$.) We note first that for this colloidal state the hard core is completely masked by the Coulomb repulsion of the surface charges. Further, the higher-order correlations become progressively weaker and shorter ranged. These features continue to hold for the correlation functions $g_{20}(r), g_{21}(r), g_{22}(r)$, seen in Fig. 4. In Figs. 5 and 6 , we display the Fourier transform of these functions in dimensionless form as $\rho \widetilde{h}_{i j}(k)$.

In contrast to the relatively slow vanishing of the $g_{i j}(r)$ coefficients with increasing $n^{\prime}$, seen in Figs. 3 and 4 , the coefficients $\gamma_{i j}(r)$, which are summed in Eq. (27), decrease significantly for each successive increase in $\max (i, j)$, ensuring rapid convergence of that summation. The first six of these for the same case of $s_{\sigma}=0.3$ are displayed in Figs. 7 and 8 .

The basic technique of expansions in orthogonal polynomials tailored to the distribution function $f(x)$ of the extra degrees of freedom $x$ in a generalized pair distribution function $g\left(r_{12}, x_{1}, x_{2}\right)$ may be readily applied to other systems, such as fluids of polarizable molecules [17,18]. For such internal degrees of freedom, the distribution function $f(x)$ will itself be subject to iterative calculation. Perhaps the most direct route between the liquid structure and the internal degrees of freedom is the first member of the Born-Green hierarchy [19],

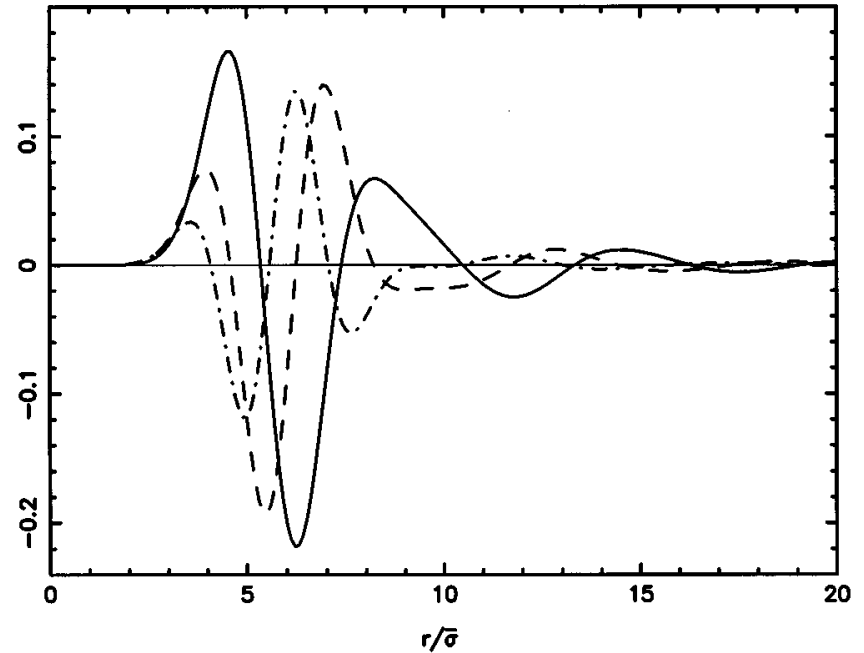

FIG. 4. Coefficients of the pair distribution function, $g\left(r, \sigma_{1}, \sigma_{2}\right)$, from the RY equation at $s_{\sigma}=0.3: g_{20}(r)$, solid line; $g_{21}(r)$, dashed line; $g_{22}(r)$, dash-dot line.

$$
\begin{aligned}
\frac{d}{d x_{1}} \ln \left[\frac{f\left(x_{1}\right)}{f_{0}\left(x_{1}\right)}\right]= & -\rho \int d \mathbf{r} d x_{2} f\left(x_{2}\right) g\left(r, x_{1}, x_{2}\right) \\
& \times \frac{\partial \beta \phi\left(r, x_{1}, x_{2}\right)}{\partial x_{1}} \\
= & -\sum_{i, j}\left[\rho \int d \mathbf{r} \sum_{k} g_{i k}(r) \beta \phi_{k j}(r)\right] \\
& \times p_{i}\left(x_{1}\right) \frac{d p_{j}\left(x_{1}\right)}{d x_{1}},
\end{aligned}
$$

where $f_{0}(x)$ is the unperturbed distribution in an isolated atom and the $p_{j}(x)$ are the orthogonal polynomials for the current $f(x)$. Recalculation of $f(x)$ from this equation will

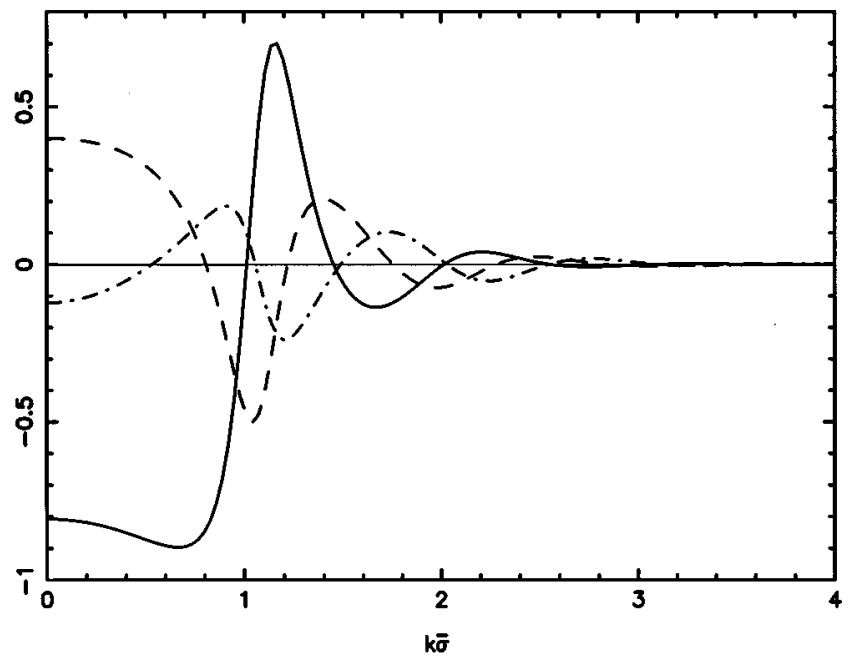

FIG. 5. Coefficients of the Fourier transform of the pair correlation function, $\rho \widetilde{h}\left(k, \sigma_{1}, \sigma_{2}\right)$, from the RY equation at $s_{\sigma}=0.3$ : $\rho \widetilde{h}_{00}(k)$, solid line; $\rho \widetilde{h}_{10}(k)$, dashed line; $\rho \widetilde{h}_{11}(k)$, dash-dot line. 


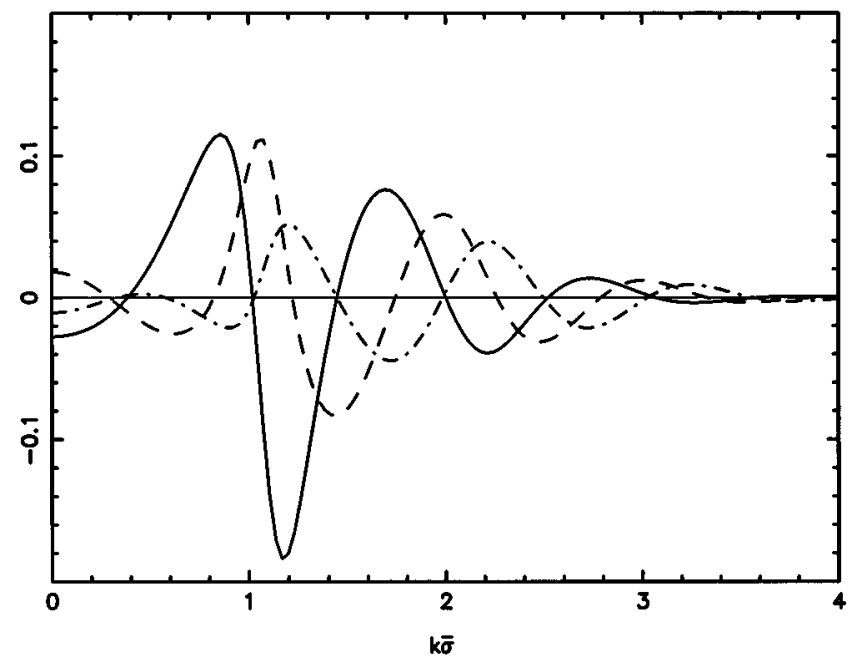

FIG. 6. Coefficients of the Fourier transform of the pair correlation function, $\rho \widetilde{h}\left(k, \sigma_{1}, \sigma_{2}\right)$, from the RY equation at $s_{\sigma}=0.3$ : $\rho \widetilde{h_{20}}(k)$, solid line; $\rho \widetilde{h_{21}}(k)$, dashed line; $\rho \widetilde{h_{22}}(k)$, dash-dot line.

then generally require a recalculation of new orthogonal polynomials, using Gram-Schmidt orthogonalization [20], for the next iteration of the structure calculation. The process continues until a self-consistent $f(x)$ is achieved.

\section{ACKNOWLEDGMENT}

I am grateful to Dr. Bruno D'Aguanno for kindly providing Monte Carlo and integral equation data from Ref. [10].

\section{APPENDIX}

An expression for the colloidal free energy in terms of correlation functions is readily obtained using the wellknown artifice of a "charging'" parameter $\xi, 0 \leqslant \xi \leqslant 1$. The following calculation is adapted from that of Morita and Hi-

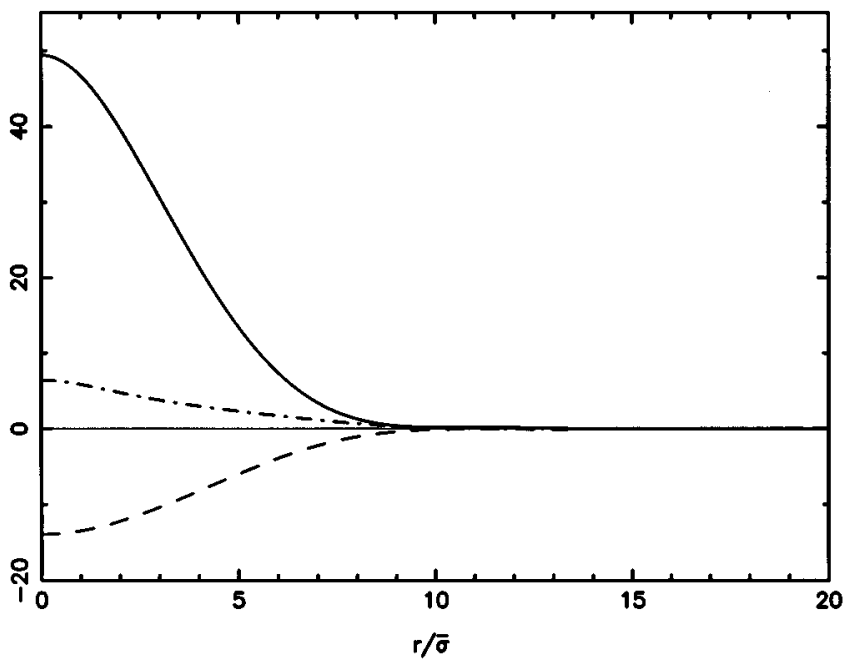

FIG. 7. Coefficients of the indirect correlation function, $\gamma\left(r, \sigma_{1}, \sigma_{2}\right)$, from the RY equation at $s_{\sigma}=0.3: \gamma_{00}(r)$, solid line; $\gamma_{10}(r)$, dashed line; $\gamma_{11}(r)$, dash-dot line.

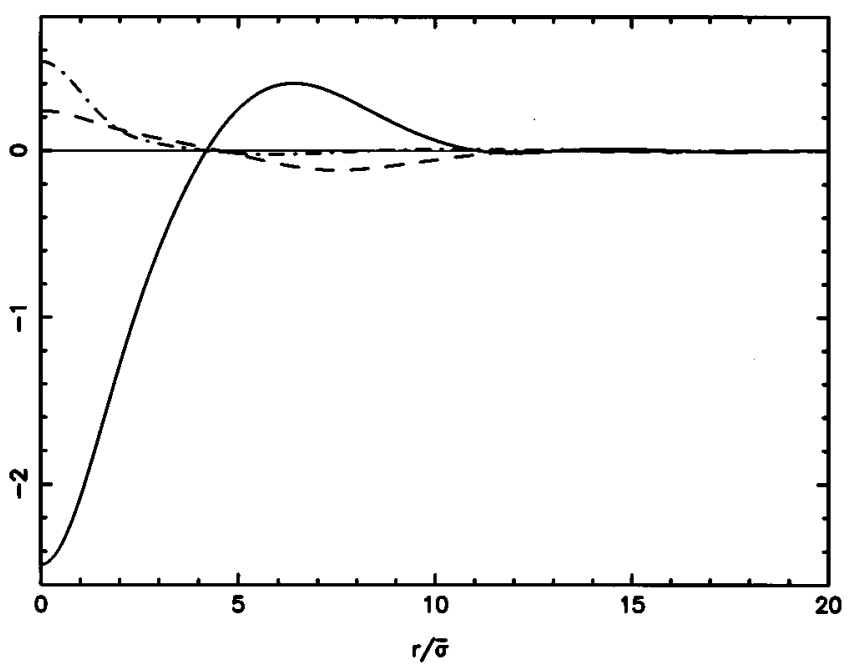

FIG. 8. Coefficients of the indirect correlation function, $\gamma\left(r, \sigma_{1}, \sigma_{2}\right)$, from the RY equation at $s_{\sigma}=0.3: \gamma_{20}(r)$, solid line; $\gamma_{21}(r)$, dashed line; $\gamma_{22}(r)$, dash-dot line.

roike [21]. Define the partition function

$$
\begin{aligned}
Q(\xi)= & \frac{1}{V^{N}} \int \prod_{j=1}^{N}\left\{d \mathbf{r}_{j} d \sigma_{j} f\left(\sigma_{j}\right)\right\} \\
& \times \exp \left[-\xi \beta \sum_{i<j} \phi\left(r_{i j}, \sigma_{i}, \sigma_{j}\right)\right]
\end{aligned}
$$

for the partially charged system. Then differentiation of $\beta A(\xi)=-\ln Q(\xi)$ gives

$$
\begin{aligned}
\frac{d \beta A(\xi)}{d \xi}= & \left\langle\sum_{i<j} \beta \phi\left(r_{i j}, \sigma_{i}, \sigma_{j}\right)\right\rangle_{\xi} \\
= & \frac{1}{2} N \rho \int d \mathbf{r} d \sigma_{1} d \sigma_{2} f\left(\sigma_{1}\right) f\left(\sigma_{2}\right) g\left(r, \sigma_{1}, \sigma_{2} ; \xi\right) \\
& \times \beta \phi\left(r, \sigma_{1}, \sigma_{2}\right),
\end{aligned}
$$

so that the excess free energy $A(\xi)$ is expressed in terms of the pair distribution function $g\left(r, \sigma_{1}, \sigma_{2} ; \xi\right)$ for the partially charged system. From Eq. (11), this function can be written

$$
\begin{aligned}
g\left(r, \sigma_{1}, \sigma_{2} ; \xi\right)= & \exp \left[-\xi \beta \phi\left(r, \sigma_{1}, \sigma_{2}\right)+\gamma\left(r, \sigma_{1}, \sigma_{2} ; \xi\right)\right. \\
& \left.+b\left(r, \sigma_{1}, \sigma_{2} ; \xi\right)\right]
\end{aligned}
$$

and so we get

$$
\begin{aligned}
\frac{\partial g\left(r, \sigma_{1}, \sigma_{2} ; \xi\right)}{\partial \xi}= & g\left(r, \sigma_{1}, \sigma_{2} ; \xi\right)\left\{-\beta \phi\left(r, \sigma_{1}, \sigma_{2}\right)\right. \\
& \left.+\frac{\partial}{\partial \xi}\left[\gamma\left(r, \sigma_{1}, \sigma_{2} ; \xi\right)+b\left(r, \sigma_{1}, \sigma_{2} ; \xi\right)\right]\right\}
\end{aligned}
$$




$$
\begin{aligned}
g\left(r, \sigma_{1}, \sigma_{2} ; \xi\right) \beta \phi\left(r, \sigma_{1}, \sigma_{2}\right) & \\
= & -\frac{\partial}{\partial \xi}\left[h\left(r, \sigma_{1}, \sigma_{2} ; \xi\right)+\frac{1}{2} h^{2}\left(r, \sigma_{1}, \sigma_{2} ; \xi\right)-g\left(r, \sigma_{1}, \sigma_{2} ; \xi\right) \gamma\left(r, \sigma_{1}, \sigma_{2} ; \xi\right)\right]+c\left(r, \sigma_{1}, \sigma_{2} ; \xi\right) \frac{\partial h\left(r, \sigma_{1}, \sigma_{2} ; \xi\right)}{\partial \xi} \\
& +g\left(r, \sigma_{1}, \sigma_{2} ; \xi\right) \frac{\partial b\left(r, \sigma_{1}, \sigma_{2} ; \xi\right)}{\partial \xi} .
\end{aligned}
$$

The left-hand side of (A5) is just the integrand of Eq. (A2); substitution into (A2) yields

$$
\begin{aligned}
\frac{\beta A}{N}= & -\frac{1}{2} \rho \int d \mathbf{r} d \sigma_{1} d \sigma_{2} f\left(\sigma_{1}\right) f\left(\sigma_{2}\right)\left[h\left(r, \sigma_{1}, \sigma_{2}\right)+\frac{1}{2} h^{2}\left(r, \sigma_{1}, \sigma_{2}\right)-g\left(r, \sigma_{1}, \sigma_{2}\right) \gamma\left(r, \sigma_{1}, \sigma_{2}\right)\right] \\
& +\frac{1}{2} \rho \int d \mathbf{r} d \sigma_{1} d \sigma_{2} f\left(\sigma_{1}\right) f\left(\sigma_{2}\right) \int_{0}^{1} d \xi c\left(r, \sigma_{1}, \sigma_{2} ; \xi\right) \frac{\partial h\left(r, \sigma_{1}, \sigma_{2} ; \xi\right)}{\partial \xi} \\
& +\frac{1}{2} \rho \int d \mathbf{r} d \sigma_{1} d \sigma_{2} f\left(\sigma_{1}\right) f\left(\sigma_{2}\right) \int_{0}^{1} d \xi g\left(r, \sigma_{1}, \sigma_{2} ; \xi\right) \frac{\partial b\left(r, \sigma_{1}, \sigma_{2} ; \xi\right)}{\partial \xi} .
\end{aligned}
$$

The second term in this expression can be integrated in Fourier transform representation as follows. From Eq. (25), it is easy to show that the matrices $\widetilde{H}$ and $\widetilde{C}$ satisfy

$$
[I+\rho \widetilde{H}(k ; \xi)]^{-1}=I-\rho \widetilde{C}(k, \xi)
$$

Then, using the matrix identity $d \operatorname{lndet} M / d \xi=\operatorname{tr}\left[M^{-1} d M / d \xi\right]$ for matrix $M$, we have

$$
\frac{\partial}{\partial \xi} \operatorname{lndet}[I+\rho \widetilde{H}(k ; \xi)]=\operatorname{tr}\left\{[I-\rho \widetilde{C}(k ; \xi)] \rho \frac{\partial \widetilde{H}(k ; \xi)}{\partial \xi}\right\},
$$

and thus

$$
\operatorname{tr}\left[\rho^{2} \widetilde{C}(k ; \xi) \frac{\partial \widetilde{H}(k ; \xi)}{\partial \xi}\right]=-\frac{\partial}{\partial \xi}\{\operatorname{lndet}[I+\rho \widetilde{H}(k ; \xi)]-\operatorname{tr}[\rho \widetilde{H}(k ; \xi)]\}
$$

But the second term in Eq. (A6) can be written

$$
\begin{aligned}
& \frac{1}{2} \rho \int d \mathbf{r} d \sigma_{1} d \sigma_{2} f\left(\sigma_{1}\right) f\left(\sigma_{2}\right) \int_{0}^{1} d \xi c\left(r, \sigma_{1}, \sigma_{2} ; \xi\right) \frac{\partial h\left(r, \sigma_{1}, \sigma_{2} ; \xi\right)}{\partial \xi} \\
& \quad=\frac{1}{2} \rho \int \frac{d \mathbf{k}}{(2 \pi)^{3}} d \sigma_{1} d \sigma_{2} f\left(\sigma_{1}\right) f\left(\sigma_{2}\right) \int_{0}^{1} d \xi \widetilde{c}\left(k, \sigma_{1}, \sigma_{2} ; \xi\right) \frac{\partial \widetilde{h}\left(k, \sigma_{1}, \sigma_{2} ; \xi\right)}{\partial \xi} \\
& \quad=\frac{1}{2} \rho \int \frac{d \mathbf{k}}{(2 \pi)^{3}} \int_{0}^{1} d \xi \sum_{i, j} \widetilde{c_{i j}}(k ; \xi) \frac{\partial \widetilde{h_{i j}}(k ; \xi)}{\partial \xi}=\frac{1}{2 \rho} \int \frac{d \mathbf{k}}{(2 \pi)^{3}} \int_{0}^{1} d \xi \operatorname{tr}\left[\rho^{2} \widetilde{C}(k ; \xi) \frac{\partial \tilde{H}(k ; \xi)}{\partial \xi}\right] .
\end{aligned}
$$

Thus, with (A9) and (A10), Eq. (A6) becomes

$$
\begin{aligned}
\frac{\beta A}{N}= & -\frac{1}{2} \rho \int d \mathbf{r} d \sigma_{1} d \sigma_{2} f\left(\sigma_{1}\right) f\left(\sigma_{2}\right)\left\{c\left(r, \sigma_{1}, \sigma_{2}\right)+\frac{1}{2}\left[c^{2}\left(r, \sigma_{1}, \sigma_{2}\right)-\gamma^{2}\left(r, \sigma_{1}, \sigma_{2}\right)\right]\right\} \\
& -\frac{1}{2 \rho} \int \frac{d \mathbf{k}}{(2 \pi)^{3}}\{\operatorname{lndet}[I+\rho \widetilde{H}(k)]-\operatorname{tr}[\rho \widetilde{H}(k)]\}+\frac{1}{2} \rho \int d \mathbf{r} d \sigma_{1} d \sigma_{2} f\left(\sigma_{1}\right) f\left(\sigma_{2}\right) \int_{0}^{1} d \xi g\left(r, \sigma_{1}, \sigma_{2} ; \xi\right) \frac{\partial b\left(r, \sigma_{1}, \sigma_{2} ; \xi\right)}{\partial \xi},
\end{aligned}
$$

after substitution of $h=\gamma+c$ in the first term; orthogonal polynomial expansions of the remaining functions of $\sigma$ finally yields Eq. (38). 
[1] P. N. Pusey, in Liquids, Freezing and the Glass Transition, edited by J.-P. Hansen, D. Levesque, and J. Zinn-Justin (North Holland, Amsterdam, 1991).

[2] J.-P. Hansen, J. Phys. Condens. Matter 5, B117 (1993).

[3] B. D’Aguanno, R. Klein, J. M. Méndez Alcaraz, and G. Nägele, in Complex Fluids, edited by L. Garrido (Springer, Berlin, 1993).

[4] L. Blum and G. Stell, J. Chem. Phys. 71, 42 (1979); 72, 2212 (1980).

[5] A. Vrij, J. Chem Phys. 71, 3267 (1979).

[6] W. L. Griffith, R. Triolo, and A. L. Compere, Phys. Rev. A 35, 2200 (1987).

[7] H. Löwen, J.-N. Roux, and J.-P. Hansen, J. Phys. Condens. Matter 3, 997 (1991).

[8] J. B. Hayter and J. Penfold, Mol. Phys. 42, 109 (1981).

[9] J.-P. Hansen and J. B. Hayter, Mol. Phys. 46, 651 (1982).

[10] B. D’Aguanno and R. Klein, Phys. Rev. A 46, 7652 (1992).
[11] B. D'Aguanno and R. Klein, J. Chem. Soc. Faraday Trans. 87, 379 (1991).

[12] J.-P. Hansen and I. R. McDonald, Theory of Simple Liquids (Academic, London, 1986).

[13] N. I. Akhiezer, The Classical Moment Problem (Hafner, New York, 1965), p. 22.

[14] G. V. Schulz, Z. Phys. Chem. Abt. B 43, 25 (1939).

[15] F. J. Rogers and D. A. Young, Phys. Rev. A 30, 999 (1984).

[16] B. D'Aguanno (private communication).

[17] J. S. Høye and G. Stell, J. Chem. Phys. 73, 461 (1980).

[18] L. R. Pratt, Mol. Phys. 40, 347 (1980).

[19] Liquids with internal degrees of freedom are discussed in detail by R. M. Stratt, Adv. Chem. Phys. 78, 1 (1990); see p. 45.

[20] G. Arfken, Mathematical Methods for Physicists (Academic, Orlando, 1985).

[21] T. Morita and K. Hiroike, Prog. Theor. Phys. 23, 1003 (1960). 